\section{FedUni ResearchOnline}

\section{https://researchonline.federation.edu.au}

Copyright Notice

This is the peer-reviewed version of the following article:

Nguyen, L., \& Valls Miro, J. (2020). An Efficient 3-Dimensional Model for Remaining Wall Thicknesses of Cast Iron Pipes in Non-Destructive Testing. IEEE Sensors Letters, 4(7), 1-1.

Which has been published in final form at:

https://doi.org/10.1109/LSENS.2020.2999330

Copyright $\odot 2020$ IEEE. Personal use of this material is permitted. Permission from IEEE must be obtained for all other uses, in any current or future media, including reprinting/republishing this material for advertising or promotional purposes, creating new collective works, for resale or redistribution to servers or lists, or reuse of any copyrighted component of this work in other works. 


\title{
An Efficient 3-Dimensional Model for Remaining Wall Thicknesses of Cast Iron Pipes in Non-Destructive Testing
}

\author{
Linh Nguyen ${ }^{1 *}$, and Jaime Valls Miro ${ }^{*}$ \\ 1 School of Science, Engineering and Information Technology, Federation University Australia, VIC 3842, Australia \\ 2 Centre for Autonomous Systems, University of Technology Sydney, Ultimo, NSW 2007, Australia \\ *Member, IEEE
}

\begin{abstract}
Over 50 percent of global pipes have been made of cast iron and most of them are ageing. In order to effectively estimate possibilities of their failures, which is paramount for efficiently managing asset infrastructures, it requires the remaining wall thickness (RWT) of a pipe to be known. In fact, RWT of the cast iron pipes can be primarily measured by the magnetism based non-destructive testing technologies though they are quite slow. To speed up the inspection process, it is proposed to sense RWT of a part of a pipe and then employ a model to predict RWT in the rest. Thus, this letter introduces a 3-dimensional (3D) model to efficiently represent RWT of a pipe given measurements collected intermittently on the pipe's surface. The proposed model first transforms 3D cylindrical coordinates to 3D Cartesian coordinates before modelling RWT by Gaussian processes (GP). The transformation allows GP to work properly on RWT data gathered on a cylindrical pipe and effectively predict RWT at unmeasured locations. Moreover, periodicity of RWT along circumference of the pipe is naturally integrated. The effectiveness of the proposed approach is demonstrated by implementation in two real-life in-service ageing cast iron pipes, where the obtained results are highly promising.
\end{abstract}

Index Terms-Sensor applications, 3D model, Gaussian process, non-destructive testing/evaluation, cast iron pipes, remaining wall thickness.

\section{INTRODUCTION}

Cast iron has been widely employed to industrially fabricate pipes for effectively transporting and distributing power generation, oil, gas, chemical and water, as accounting for over 50 percent of global pipes [1]. In Australia, older critical water pipes are primarily made of cast iron material [2]. Since most of these buried assets might have been laid over 100 years ago, accurately understanding and fully estimating possibilities of their failures in the pipeline network are paramount for efficiently managing the asset infrastructures by utility companies, which provides better renewal programs, break reduction, customer satisfaction or minimal replacement costs [3]. Many approaches have been proposed to assess the failures of the pipes, particularly water mains, as summarised in [4]. One of premises behind estimating whether a pipe will fail in the near future or not is that the remaining wall thickness (RWT) of the pipe is required to be known [5]-[7]. For instance, a thickness section of a $600 \mathrm{~mm}$ diameter cast iron water pipe excavated in Sydney is shown in Fig. 1, where a critically low remaining wall thickness patch is apparently visible.

In order to conduct measurements of the cast iron pipe thickness, the in-service testing methods, also known as Non-Destructive Testing (NDT) or Evaluation (NDE) are extensively exploited for evaluating the RWT of the cast iron pipes in industry [8]. Among those techniques, the pulse Eddy current (PEC) technology presents multiple advantages as demonstrated in our previous works [9]-[11]. Nevertheless, not only the PEC, most of the NDT/NDE approaches are derived from the principle of magnetism in which the magnetic field takes time to penetrate through the cast iron material (e.g. methods including Eddy current, remote field Eddy current and PEC) or magnetize the pipe (e.g. magnetic flux leakage) [9]. In other words, scanning the RWT of the cast iron mains by the magnetism based technologies is deemed to be quite slow, particularly when applied for a long

Corresponding author: Linh Nguyen (e-mail: I.nguyen@federation.edu.au).

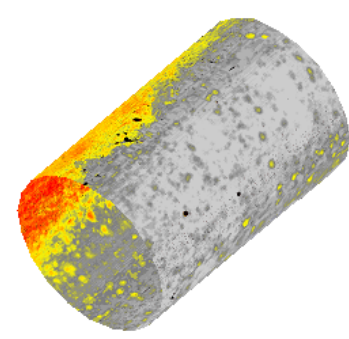

(a)

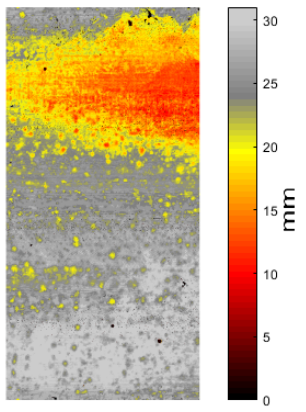

(b)
Fig. 1: Water pipe thickness interpretations in (a) 3D and (b) 2D.

pipeline. Moreover, it may cause discontinuity of supply to customers while the NDT/NDE sensors are scanning the cast iron thickness of a pipe still providing service. For instance, in our previous work [12], an autonomous tool has been designed to internally evaluate RWT of a cast iron main. Nonetheless, it requires the main to be dewatered before the tool is deployed. That is, scanning the pipe thickness can only be implemented in a short time (e.g. few hours) during the shutdown of the water main. Therefore, in order to maximally extend a section of a pipe to be evaluated, in the work [12], we have proposed to intermittently take measurements. In equivalent words, the autonomous tool collects measurements on one ring along circumference of the pipe, skips another ring and repeats the process. And ultimately, given the collective measurements, the unmeasured area can be filled up by inference if a model of the pipe thicknesses is available.

In literature, some methods have been proposed to model RWT presenting on a cylindrical pipe. As demonstrated in Fig. 1, the cast iron pipe thickness can be 3-dimensionally represented or 2-dimensionally unwrapped. In the works [1], [13], the authors proposed to model RWT of a cast iron pipe in simulation by the finite element analysis technique. Nevertheless, the proposed model is computationally 
complicated and not able to predict RWT at unmeasured areas on the pipe. Moreover, Zhang et al. in [14] introduced a Gaussian process (GP) based method to model an arch tunnel's inner surface profile. They proposed to transform a 3-dimensional (3D) coordinate in the arch tunnel into a 2-dimensional (2D) coordinate before modelling the surface profile by GP. However, periodicity of the thickness/profile along circumference, which appears in the pipe but not in the arch tunnel, has not been considered yet. In our previous work [12], a 2D model based on GP for RWT of a cast iron pipe was taken into account. It was proposed to employ a periodic function [15] to incorporate into a covariance function to construct a periodic kernel for the model. It is noted that since the periodic function is generic, the proposed model computes periodicity in both circumferential and longitudinal directions of a cylindrical pipe though considering periodicity in the longitudinal direction is not necessary.

Therefore, in this paper, by extending the previous works, we propose a GP based 3D model to represent RWT of a pipe. The proposed model first transforms a cylindrical coordinate on the pipe to a 3D Cartesian coordinate before modelling RWT by GP. The transformation allows GP to work properly on RWT data gathered on a cylindrical pipe and effectively predict RWT at unmeasured locations. Moreover, periodicity of RWT along circumference of the pipe is naturally incorporated. Particularly, we have validated the proposed model in the real-life RWT dataset collected on the water mains in Sydney, Australia with highly promising results.

\section{3-DIMENSIONAL MODEL FOR REMAINING WALL THICKNESSES OF PIPES}

To model RWT of a pipe by the use of GP [15], it is assumed that only one thickness value is measured underneath a sensor footprint and the coordinate of centroid of the sensor footprint presents the location of the corresponding thickness measurement. Hence, on the pipe's surface, one has point measurements and their corresponding locations. On the other hand, locations on the pipe's surface are normally presented in a cylindrical coordinate system. That is, a point on a cylindrical pipe's surface can be coordinated by three parameters including radial distance $\rho$, angular coordinate $\theta$ and height $z$. For the cast iron pipes used in water mains, if $\rho$ is constant along the pipeline, which gets the length scale hyperparameter of the GP model learned to be zero. Furthermore, in the case two locations with $\theta=1^{\circ}$ and $\theta=360^{\circ}$ given the same other coordinates, the GP model treats measurements at those locations far away in the circumferential direction though they are adjacent in reality due to closeness of the pipe. Thus, to guarantee the GP model to work properly on modelling RWT of the pipe, we propose to transform a $3 \mathrm{D}$ cylindrical coordinates to a $3 \mathrm{D}$ Cartesian coordinate as follows.

$$
p=(x, y, z)=(\rho \cos \theta, \rho \sin \theta, z)
$$

where $p=(x, y, z)$ is the 3D Cartesian coordinate. Note that $\rho$ is not necessarily constant since the internal and external corrosion varies along the pipe.

If we denote the RWT collected at the location $p_{i}$ as $t_{i}$, then all the $n$ RWT measurements on the pipe can be modelled by

$$
\boldsymbol{t}(\boldsymbol{p})=\boldsymbol{v}(\boldsymbol{p})+\varepsilon,
$$

where $\boldsymbol{p}=\left(p_{1}^{T}, p_{2}^{T}, \cdots, p_{n}^{T}\right)^{T} \in \mathbb{R}^{n \times 3}, \boldsymbol{t}=\left(t_{1}, t_{2}, \cdots, t_{n}\right)^{T} \in \mathbb{R}^{n}$ and $\boldsymbol{v}=\left(v_{1}, v_{2}, \cdots, v_{n}\right)^{T} \in \mathbb{R}^{n}$ is the latent variables at $\boldsymbol{p}$. $\varepsilon=$ $\left(\epsilon_{1}, \epsilon_{2}, \cdots \epsilon_{n}\right) \in \mathbb{R}^{n}$, where $\epsilon_{i}$ is a measurement noise with a zero mean and an unknown variance $\sigma_{n}^{2}$. In this work, the latent variables $\boldsymbol{v}$ are proposed to be modelled by GP, which is specified by the mean $\mu$ and covariance $C$ functions. For the purpose of simplicity, we assume the mean function to be a constant as averaging all the RWT measurements. There is a rich family of covariance functions for the GP model [15]. Of which the automatic relevance determination Matern kernel will be used for demonstration in this work, as given below.

$$
C\left(p_{i}, p_{j} \mid \Theta\right)=\sigma^{2}(1+\sqrt{3} d) \exp (-\sqrt{3} d)
$$

presents correlation between the RWT values at locations $p_{i}$ and $p_{j}$, where $\sigma$ is the RWT standard deviation, and

$$
d=\sqrt{\sum_{k=1}^{2} \frac{\left(p_{i}-p_{j}\right)^{T}\left(p_{i}-p_{j}\right)}{l_{k}^{2}}},
$$

where $l_{k}$ is the characteristic length scale in the $k$ direction. Note that in modelling RWT of a pipe, due to corrosion processes [16], two any RWT values have spatially correlated influence on each other in both the circumferential and longitudinal directions, respectively. $\Theta=\left(\sigma, l_{1}, l_{2}, \sigma_{n}\right)$ is a set of hyperparameters. These hyperparameters can be estimated from the collective RWT measurements by utilizing the maximum likelihood technique [17].

In terms of inference, given the GP model learned from the RWT measurements $\boldsymbol{t}(\boldsymbol{p})$, we can predict RWT at $m$ unmeasured locations $\boldsymbol{p}^{*}$ on the pipe, as presented in the formulations of their posterior means $m_{p^{*}}$ and covariances $\Sigma_{p^{*}}$

$$
\begin{gathered}
m_{p^{*}} \mid \boldsymbol{t}(\boldsymbol{p})=\mu\left(\boldsymbol{p}^{*}\right)+\Sigma_{\boldsymbol{p} \boldsymbol{p}^{*}}^{T}\left(\Sigma_{\boldsymbol{p p}}+\sigma_{n}^{2} I\right)^{-1}(\boldsymbol{t}(\boldsymbol{p})-\mu(\boldsymbol{p})) \\
\Sigma_{\boldsymbol{p}^{*}} \mid \boldsymbol{t}(\boldsymbol{p})=\Sigma_{\boldsymbol{p}^{*} \boldsymbol{p}^{*}}-\Sigma_{\boldsymbol{p} \boldsymbol{p}^{*}}^{T}\left(\Sigma_{\boldsymbol{p} \boldsymbol{p}}+\sigma_{n}^{2} I\right)^{-1} \Sigma_{\boldsymbol{p} \boldsymbol{p}^{*}}
\end{gathered}
$$

where $\mu\left(\boldsymbol{p}^{*}\right)(\mu(\boldsymbol{p}))$ and $\Sigma_{\boldsymbol{p}^{*} \boldsymbol{p}^{*}}\left(\Sigma_{\boldsymbol{p} \boldsymbol{p}}\right)$ are a $m(n)$ RWT mean vector and a $m \times m(n \times n)$ covariance matrix at locations $\boldsymbol{p}^{*}(\boldsymbol{p})$, respectively. $\Sigma_{p p^{*}}$ is a $n \times m$ covariance matrix presenting correlation between the RWT values at locations $\boldsymbol{p}^{*}$ and $\boldsymbol{p}$ while $I$ is a $n \times n$ identity matrix. Note that components of the covariance matrices are computed by the use of the covariance function (3).

\section{EXPERIMENTS AND RESULTS}

To validate effectiveness of the proposed model, we conducted experiments on the real-life in-service water mains along Hector street in Sydney, Australia from November to December 2017 by digging the holes $(3.6 \mathrm{~m} \times 2 \mathrm{~m} \times 2 \mathrm{~m})$ around the pipes and employing the PEC sensor [9]-[12] to inspect RWT of the 1922 ageing cast iron pipes. The sensor fully scanned 9 section pipes, ranging from $1.7 \mathrm{~m}$ to $3.1 \mathrm{~m}$, at different locations along the pipelines at resolution of 50 $\mathrm{mm} \times 50 \mathrm{~mm}$, which compromises between the scanning speed and measurement accuracy. That is, the pipe's surface was discretized into a grid, where a grid cell was sized by $50 \mathrm{~mm} \times 50 \mathrm{~mm}$. The PEC sensor's footprint covered the grid cell at each scan, and only one RWT measurement was reported at a corresponding grid cell. Some RWT maps of the fully scanned cast iron pipes are demonstrated in Figures 2a, 2b and 2c, where the longitudinal direction is along the horizontal axis while the circumferential direction is along the vertical axis after the $3 \mathrm{D}$ closed pipe is unwrapped.

In order to verify how the proposed model presents RWT of a pipe, we considered the RWT measurements of the fully scanned pipes as ground truths. For the RWT data of each pipe, a part of 


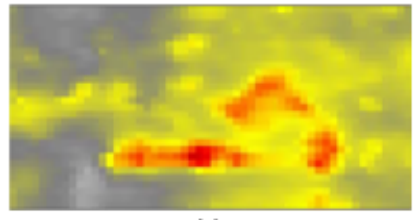

(a)

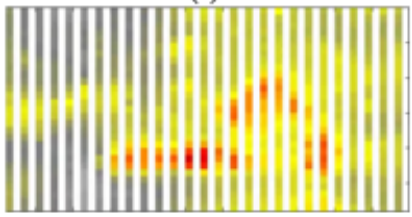

(d)

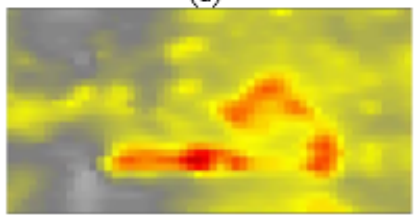

(g)

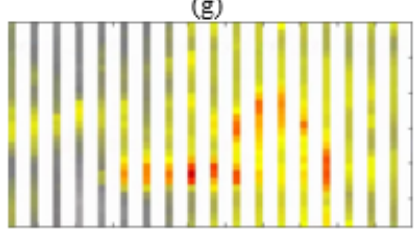

(j)

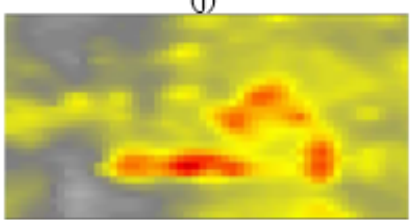

(m)

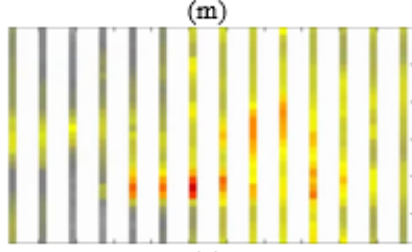

(p)

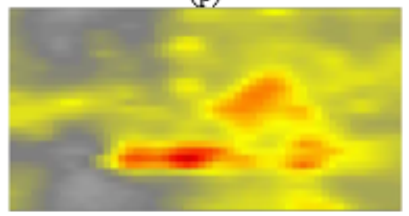

(s)

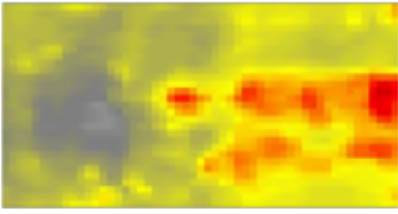

(b)

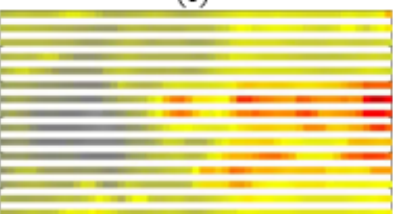

(e)

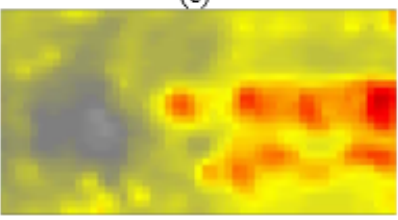

(h)

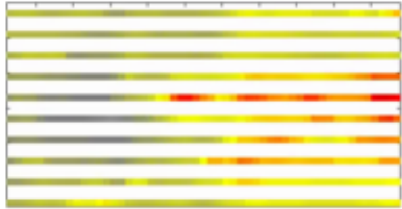

(k)

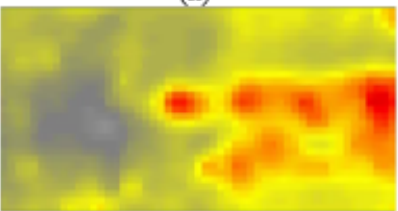

(n)

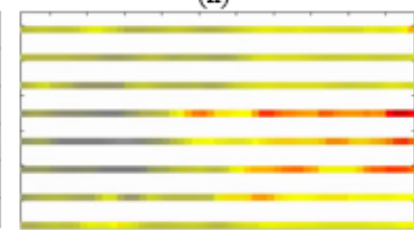

(q)

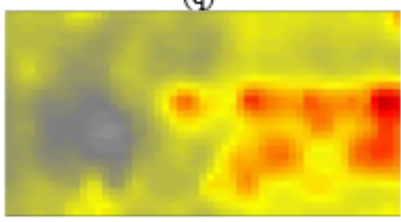

(t)

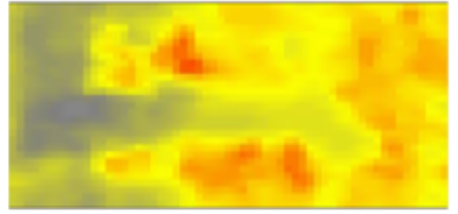

(c)

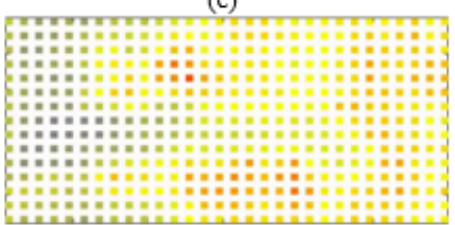

(f)

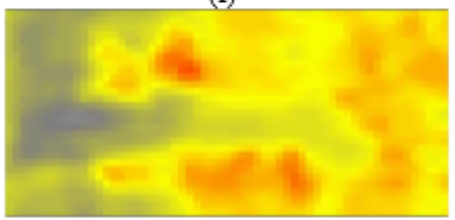

(i)

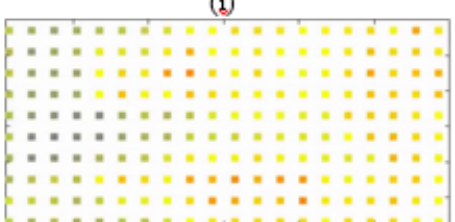

(1)

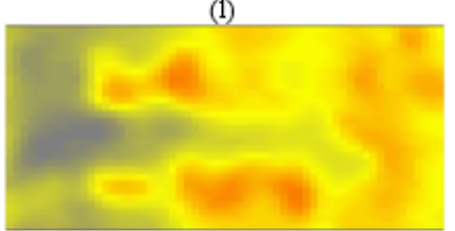

(o)

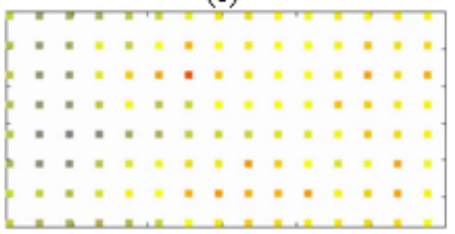

(r)

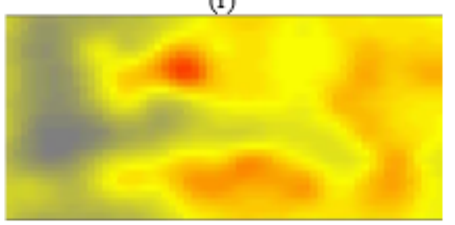

(u)

Fig. 2: Experimental results: (a), (b) and (c): ground truths of 3 different pipes; (d), (e), (f), (j), (k), (l), (p), (q) and (r): possibilities of operating the scan, where there is no scanning in white areas; $(\mathrm{g}),(\mathrm{h}),(\mathrm{i}),(\mathrm{m}),(\mathrm{n}),(\mathrm{o}),(\mathrm{s}),(\mathrm{t})$ and $(\mathrm{u})$ : partly scanned RWT maps are filled up by inferences.

them was used for training the model, and the remaining was used for testing. Based on our previous work [12], it is supposed that there are 3 possibilities of operating the scanning to speed up the inspection process, where there is no scanning in white areas: (1) the sensor circumferentially scans one ring and then skips one or more rings, as demonstrated in Figures $2 \mathrm{~d}, 2 \mathrm{j}$ and $2 \mathrm{p}$; (2) the sensor longitudinally scans one line and then skips one or more lines, as shown in Figures 2e, 2k and 2q; (3) the sensor scans one grid cell and then skips one or more grid cells, as illustrated in Figures 2f, 21 and 2r. For the purpose of demonstrations, we skipped one, two and three $\operatorname{ring}(\mathrm{s}) /$ line(s)/grid(s) in the experiments as shown in Fig. 2. Upon these configurations, for each pipe the models were trained by using the RWT measurements at locations of which the sensor could scan. The learned models were then employed to predict the RWT values at unscanned locations; and the filled RWT maps are shown in Figures $2 \mathrm{~g}, 2 \mathrm{~h}, 2 \mathrm{i}, 2 \mathrm{~m}, 2 \mathrm{n}, 2 \mathrm{o}, 2 \mathrm{~s}, 2 \mathrm{t}$ and $2 \mathrm{u}$, correspondingly. It is noted that in Fig. 2 each filled RWT map is corresponded to the possible scanning configuration immediately right on its top. For instance, Fig. $2 \mathrm{~g}$ was created after filling up the white areas in Fig. $2 \mathrm{~d}$ by the predictions interpolated by the model trained from the collected RWT data as illustrated in Fig. 2d. Qualitatively, the filled maps in each column of Fig. 2 are highly comparable to the ground truths demonstrated in Figures $2 \mathrm{a}, 2 \mathrm{~b}$ and $2 \mathrm{c}$, respectively. More importantly, by reducing the number of measurements needed, 


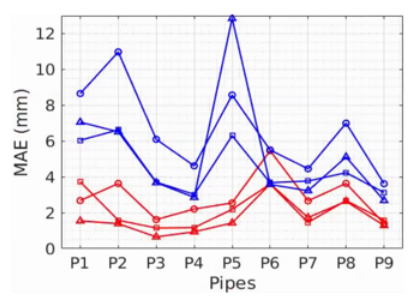

(a)

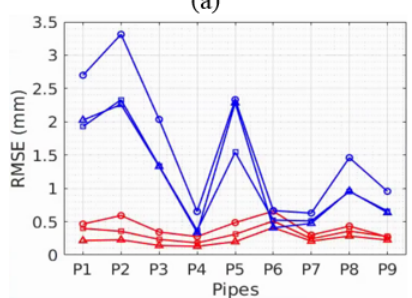

(d)

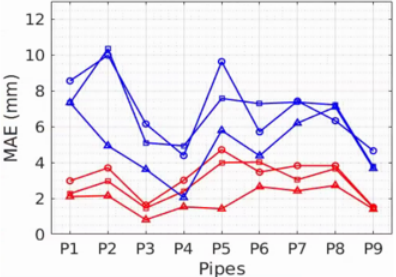

(b)

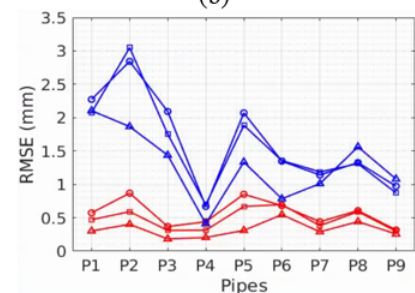

(e)

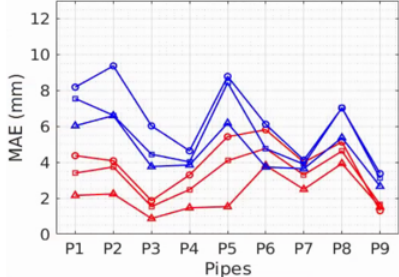

(c)

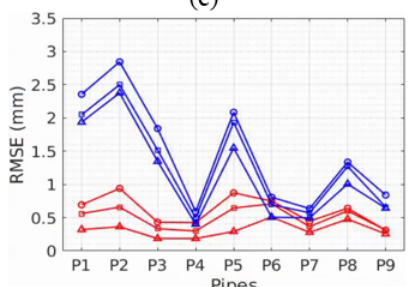

(f)

$\triangle-3 D-1$ line/cell break $\rightarrow-3 D-2$ line/cell break $\rightarrow-3 D-3$ line/cell break $\rightarrow-2 D-1$ line/cell break $\rightarrow-2 D-2$ line/cell break $\rightarrow-2 D \cdot 3$ line/cell break

Fig. 3: Maximum absolute errors and root mean square errors for 3 possible sensing configurations. (a) and (d): 1, 2 and 3 ring skip/break; (b) and (e): 1, 2 and 3 line skip/break; (c) and (f): 1, 2 and 3 grid cell skip/break.

scanning time is significantly declined. For instance, if we use the sensing strategies presented in the second, fourth and sixth rows of Fig. 2, the scanning time is reduced to $50 \%, 67 \%$ and $75 \%$, respectively.

Moreover, to quantitatively evaluate the proposed model, we computed maximum absolute errors (MAE) and root mean square errors (RMSE) between the ground truths and their counterpart predictions for 3 possible scanning configurations in each scanned pipe, as demonstrated in Fig. 3. For the purpose of comparisons, we also implemented the 2D GP model [15] with a given periodic function in the dataset of 9 scanned pipes. It can be apparently seen in Fig. 3 that the proposed model outperforms the 2D GP model at any possible sensing configurations. Moreover, as expected, the more RWT measurements are collected, the smaller MAE and RMSE can be obtained.

\section{CONCLUSIONS}

The letter has proposed an efficient 3D model for modelling RWT of a cast iron pipe to speed up the sensing process in the NDT applications. The proposed model is based on GP, where 3D cylindrical coordinates of a point on the pipe are transformed to 3D Cartesian coordinates, which enables GP to work properly. The transformation also allows the model to naturally incorporate periodicity of RWT along circumference of a pipe. Furthermore, correlation between any two RWT values is interpreted in two circumferential and longitudinal directions due to natural corrosion process. We have extensively validated the model in the realistic datasets collected on two inservice ageing cast iron pipes.

\section{ACKNOWLEDGMENT}

This work is an outcome from the Operationalisation project funded by Sydney Water Corporation.

\section{REFERENCES}

[1] C. Zhang, S. Rathnayaka, B. Shannon, J. Ji, and J. Kodikara, "Numerical interpretation of pressurized corroded cast iron pipe tests," International Journal of Mechanical Sciences, vol. 128-129, pp. 116-124, 2017.
[2] J. VallsMiro, J. Rajalingam, T. Vidal-calleja, F. Bruijn, T. Wood, D. Vitanage, N. Ulapane, B. Wijerathna, and D. Su, "A live test-bed for the advancement of condition assessment and failure prediction research on critical pipes," Water Asset Management International, vol. 10(2), pp. 03-08, 2014.

[3] D. Vitanage, J. Kodikara, and G. Allen, "Collaborative research on condition assessment and pipe failure prediction for critical water mains," Water Asset Management International, vol. 10, pp. 15-18, 2014.

[4] J. Kodikara, S. Rathnayaka, J. Zhang, C. Crawly, D. Zhang, and F. Blaha, "Lessons learned from large-diameter pipe failure case studies," in Proc. Conferece on Pipelines, Arizona, USA, August 2017, pp. 561-571.

[5] W. Wang, A. Zhou, G. Fu, C. Q. Li, D. Robert, and M. Mahmoodian, "Evaluation of stress intensity factor for cast iron pipes with sharp corrosion pits," Engineering Failure Analysis, vol. 81, pp. 254-269, 2017.

[6] A. Fahimi, T. S. Evans, J. Farrow, D. A. Jesson, M. J. Mulheron, and P. A. Smith, "On the residual strength of aging cast iron trunk mains: Physically-based models for asset failure," Materials Science \& Engineering A, vol. 663, pp. 204-212, 2016.

[7] J. Ji, D. J. Robert, C. Zhang, D. Zhang, and J. Kodikara, "Probabilistic physical modelling of corroded cast iron pipes for lifetime prediction," Structural Safety, vol. 64, pp. 62-75, 2017.

[8] Z. Liu and Y. Kleiner, "State of the art review of inspection technologies for condition assessment of water pipes," Measurement, vol. 46(1), pp. 1-15, 2013.

[9] L. Nguyen, N. Ulapane, J. V. Miro, G. Dissanayake, and F. Munoz, "Improved signal interpretation for cast iron thickness assessment based on pulsed eddy current sensing," in Proc. IEEE Conference on Industrial Electronics and Applications, 2017, pp. 2005-2010.

[10] N. Ulapane, L. Nguyen, J. V. Miro, A. Alempijevic, and G. Dissanayake, "Designing a pulsed eddy current sensing set-up for cast iron thickness assessment," in Proc. IEEE Conference on Industrial Electronics and Applications, 2017, pp. 901-906.

[11] N. Ulapane, L. Nguyen, J. V. Miro, and G. Dissanayake, "A solution to the inverse pulsed eddy current problem enabling 3d profiling," in Proc. IEEE Conference on Industrial Electronics and Applications, 2018, pp. 1267-1272.

[12] J. VallsMiro, N. Ulapane, L. Shi, D. Hunt, and M. Behrens, "Robotic pipeline wall thickness evaluation for dense nondestructive testing inspection," Journal of Field Robotics, vol. 35, pp. 1293-1310, 2018.

[13] C. Zhang, J. Ji, J. Kodikara, and B. Rajani, "Hyperbolic constitutive model to study cast iron pipes in 3-d nonlinear finite element analyses," Engineering Failure Analysis, vol. 75, pp. 26-36, 2017.

[14] C. Zhang, Y. Lei, L. Zhang, and N. Chen, "Modeling tunnel profile in the presence of coordinate errors: A Gaussian process-based approach," IISE Transactions, vol. 49(11), pp. 1065-1077, 2017.

[15] C. E. Rasmussen and C. K. I. Williams, Gaussian processes for machine learning. The MIT Press, Cambridge, Massachusetts, London, England, 2006.

[16] H. Zhu and L. M. Zhang, "Characterizing geotechnical anisotropic spatial variations using random field theory," Canadian Geotechnical Journal, vol. 50, pp. 723-734, 2013.

[17] L. Nguyen, S. Kodagoda, R. Ranasinghe, and G. Dissanayake, "Informationdriven adaptive sampling strategy for mobile robotic wireless sensor network," IEEE Transaction on Control Systems Technology, vol. 24(1), pp. 372-379, 2016. 